Ökologisch nachhaltige Strukturförderung in Baden-Württemberg

\section{Umweltbewertung von EU-Fördermaßnahmen}

Die Europäische Kommission hat das Leitbild der nachhaltigen Entwicklung zum Bestandteil ihrer Strukturpolitik der aktuellen Förderperiode gemacht. Doch wie Nachhaltigkeit bei der Projektförderung geprüft und bewertet werden soll, ließ die Kommission offen. Wie die Umweltwirkungen von Projekten bewertet werden können, zeigt ein Programm aus Baden-Württemberg. Von Dominic Kaiser

n Förderprogrammen zur Umsetzung der Strukturpolitik, die aus Strukturfonds wie dem EFRE (Europäischer Fonds für regionale Entwicklung) finanziert werden, ist die Nachhaltige Entwicklung als Querschnittsziel zu berücksichtigen. In Baden-Württemberg wurde eine Konzeption zur Nachhaltigkeitsbewertung von Projekten entwickelt, die im Rahmen des Querschnittsziels Nachhaltige Entwicklung für das Operationelle Programm „Regionale Wettbewerbsfähigkeit und Beschäftigung“ (RWB-EFRE) 2007-2013 gefördert werden.

\section{Ziele der Umweltbewertung}

Strategische Ziele des Programms sind die Stärkung und der Ausbau von Wettbewerbsfähigkeit, Beschäftigung und nachhaltiger Entwicklung in allen Teilräumen Baden-Württembergs. Um diese Ziele zu erreichen, wurden drei spezifisch ausgerichtete Förderschwerpunkte ausgewiesen. Abbildung 1 zeigt die Struktur des Operationellen Programms.

Im Rahmen des Querschnittsziels Nachhaltige Entwicklung werden sämtliche Projekte auf ihre Umweltwirkungen hin bewertet und die Ergebnisse bei der Projektauswahl berücksichtigt. Wichtigstes Ziel dieses Vorgehens ist die Steuerung der Projektauswahl nach Umweltaspekten. Projekte mit insgesamt als negativ bewerteten Umweltwirkungen werden nicht gefördert. Zur Bewertung werden im Antragsverfahren sogenannte Indikatorenformulare von den Antrag- stellern ausgefüllt und von Verwaltungsstellen, die an der Programmumsetzung beteiligt sind, ausgewertet. Eine wichtige Anforderung an die Konzeption ist, dass die Projektbewertungen mit einem im Rahmen des Antragsverfahrens vertretbaren Aufwand zu bearbeiten sind.

Weiteres Ziel der Umweltbewertung ist es, den Vergleich von Umweltwirkungen des Mitteleinsatzes in den unterschiedlichen Programmebenen, wie den Maßnahmenbereichen oder Schwerpunkten, zu ermöglichen. Eine weitere Württemberg den-Württemberg 2007-2013
Anforderung an die Bewertungskonzeption ist daher, dass die Ergebnisse auf Projektebene aggregierbar und untereinander vergleichbar sind.

\section{Konzeptionelle Grundlagen}

Bei Entwicklung der Bewertungskonzeption wurde das thematisch recht breite Spektrum der Fördermaßnahmen zunächst auf systematische Unterschiede von Umweltwirkungen hin untersucht. Diese Unterschiede werden anhand der beiden folgenden Beispiele aufgezeigt.

Um die Clusterentwicklung auf dem Gebiet der Nanotechnologie zu unterstützen, werden die Personalkosten eines Clustermanagers gefördert. Zu den Aufgaben des Managers gehört es, die Zusammenarbeit und Vernetzung relevanter Akteure, wie Unternehmen und Forschungseinrichtungen, zu intensivieren und zu koordinieren. Die Tätigkeit des Managers hat direkte Umweltwirkungen wie beispielsweise der Energieverbrauch bei Büroarbeit oder Dienstreisen. Weitere Wirkungen liegen im Bereich der Ergebnisse der Dienstleistung des Managers. So etwa, wenn in Folge der Vernet-

Abbildung 1: Ziel- und Strategiesystem des Operationellen Programms RWB-EFRE 2007-2013 in Baden-

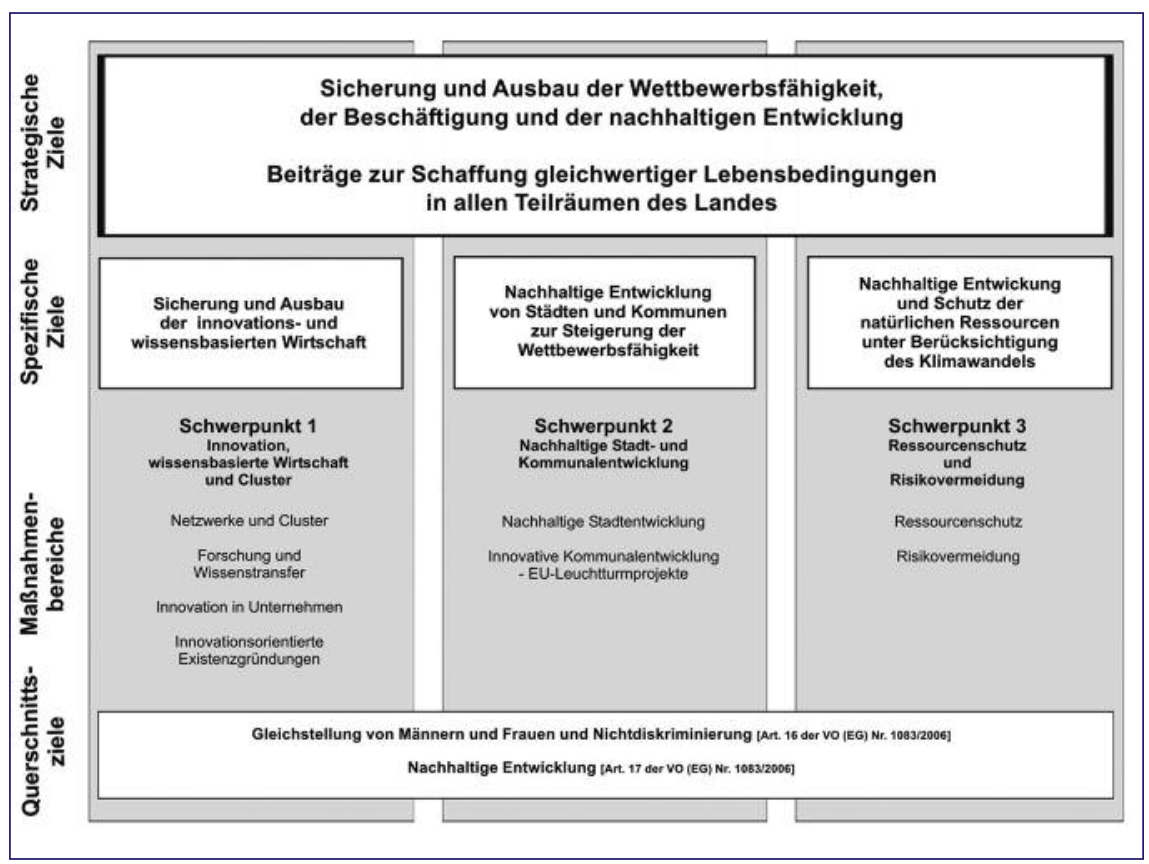

Quelle: Operationelles Programm „Regionale Wettbewerbsfähigkeit und Beschäftigung“, Teil EFRE in Ba- 
zung eines Herstellers von Nanopartikeln mit einem Automobilhersteller nanobasierte Schmiermittel entwickelt werden, die zu reduziertem Verschleiß und verlängerter Lebensdauer von Verbrennungsmotoren führen. Diese werden als indirekte Umweltwirkungen des Projekts betrachtet.

Ein weiteres Beispiel ist ein gewerbliches Unternehmen, das eine Förderung für ein neues Produktionsgebäude und Produktionsmaschinen zur Herstellung energieeffizienter Elektromotoren erhält. Umweltwirkungen des Projekts ergeben sich aus der Errichtung und Nutzung des Gebäudes und aus der Beschaffung und dem Betrieb der Maschinen, etwa in Form von Flächen-, Energie- und Materialverbräuchen und Emissionen. Diese entstehen bei Projektdurchführung und Nutzung von Gebäuden und Maschinen durch das Unternehmen und können als direkte Umweltwirkungen der Förderung bezeichnet werden. Weitere Wirkungen

Tabelle 1: Stufen der Gesamtbewertung investiver und nicht-investiver Projekte nach Umweltwirkungen

\begin{tabular}{lcccc}
\hline Ergebnis der Gesamtbewertung (Punkte) & -1 & 0 & 1 & 2 \\
\hline $\begin{array}{l}\text { Bewertung bezüglich des Beitrags zum } \\
\text { Querschnittsziel Nachhaltige Entwicklung }\end{array}$ & negativ & neutral & fördernd & $\begin{array}{c}\text { besonders } \\
\text { fördernd }\end{array}$ \\
\hline Förderung im Operationellen Programm & $\begin{array}{c}\text { nicht } \\
\text { förderfähig }\end{array}$ & förderfähig & förderfähig & förderfähig \\
\hline
\end{tabular}

Quelle: Eigene Zusammenstellung

Tabelle 2: Indikatoren zur Bewertung nicht-investiver Projekte

\begin{tabular}{ll}
\hline $\begin{array}{l}\text { Themenbereiche und } \\
\text { Indikatoren }\end{array}$ & Gegenstand der Bewertung \\
\hline 1. Ergebnisse des Projekts & $\begin{array}{l}\text { Sind von den angestrebten Ergebnissen des Vorhabens Aus- } \\
\text { wirkungen auf Interessen des Umweltschutzes zu erwarten? }\end{array}$ \\
\hline 2. Ziele des Projekts & $\begin{array}{l}\text { Sind Verbesserungen im Bereich des Umweltschutzes } \\
\text { eines der vorrangigen Vorhabensziele? }\end{array}$ \\
\hline 3. Breitenwirkung der Projektergebnisse & $\begin{array}{l}\text { Besitzen die angestrebten Ergebnisse des Vorhabens } \\
\text { Potential für die Entfaltung einer Breitenwirkung? }\end{array}$ \\
\hline 4. Projektdurchführung & $\begin{array}{l}\text { Werden bei der Durchführung des Projekts die Prinzipien } \\
\text { einer umweltfreundlichen Beschaffung eingehalten? }\end{array}$ \\
\hline Quelle: Eigene Zusammenstellung &
\end{tabular}

Tabelle 3: Themenbereiche und Indikatoren zur Umweltbewertung investiver Projekte

\begin{tabular}{|c|c|}
\hline $\begin{array}{l}\text { Themenbereiche und } \\
\text { Indikatoren }\end{array}$ & Gegenstand der Bewertung \\
\hline \multicolumn{2}{|l|}{ Ressourcenschutz } \\
\hline 1. Erneuerbare Energien & $\begin{array}{l}\text { Erhöhung des Anteils erneuerbarer Energien an der } \\
\text { Energiegewinnung }\end{array}$ \\
\hline 2. Energieeinsatz & Sparsamer und effizienter Energieeinsatz \\
\hline 3. Materialeinsatz & Sparsamer und effizienter Materialeinsatz \\
\hline 4. Flächenverbrauch & $\begin{array}{l}\text { Ersatz nicht-erneuerbarer durch erneuerbare Rohstoffe } \\
\text { Art und Umfang der Flächennutzung des Projekts }\end{array}$ \\
\hline 5. Schutzgut Wasser & $\begin{array}{l}\text { Auswirkungen auf das Schutzgut Wasser, sparsamer } \\
\text { und effizienter Umgang mit Wasser }\end{array}$ \\
\hline 6. Schutzgut Luft & Auswirkungen auf das Schutzgut Luft \\
\hline \multicolumn{2}{|l|}{$\begin{array}{l}\text { Weitere umweltrelevante Aspekte } \\
\text { von Vorhaben }\end{array}$} \\
\hline 7. Biologische Vielfalt, Fauna und Flora & $\begin{array}{l}\text { Auswirkungen auf geschützte Gebiete und die Tier- und } \\
\text { Pflanzenwelt }\end{array}$ \\
\hline 8. Verkehr & Auswirkungen des Vorhabens auf den Verkehr \\
\hline 9. Multiplikatorcharakter & $\begin{array}{l}\text { Übertragbarkeit positiver Umweltwirkungen auf ähnliche } \\
\text { Anwendungsfälle }\end{array}$ \\
\hline 10. Weitere Umweltwirkungen & $\begin{array}{l}\text { Wertung einer weiteren, mit übrigen Fragen nicht } \\
\text { berücksichtigten Wirkung }\end{array}$ \\
\hline \multicolumn{2}{|l|}{ Umweltmanagement } \\
\hline 11. Umweltmanagementsysteme & $\begin{array}{l}\text { Umwelt-, Energie- oder Stoffstrommanagement im } \\
\text { geförderten Unternehmen }\end{array}$ \\
\hline 12. Umwelt-Produktzeichen & $\begin{array}{l}\text { Herstellung von Produkten mit Umweltzeichen } \\
\text { einer umweltfreundlichen Beschaffung eingehalten? }\end{array}$ \\
\hline
\end{tabular}

Quelle: Eigene Zusammenstellung treten bei Einsatz der hergestellten Elektromotoren durch Kunden des Unternehmens auf. Da sie nur mittelbar in Verbindung mit den Investitionsobjekten entstehen, werden sie als indirekte Wirkungen der Projektförderung betrachtet.

Für die Gesamtwirkungen des Clustermanagements sind die direkten Umweltwirkungen aus der Tätigkeit des Clustermanagers gegenüber den potenziellen indirekten Wirkungen nur marginal. Es erscheint daher nicht zielführend, die Förderfähigkeit eines solchen Projekts auf die direkten Umweltwirkungen zu stützen.

Im Gegensatz dazu führt die Förderung des Unternehmens über die Investitionen in Gebäude und Maschinen zu konkreten, zeitlich und räumlich klar abgrenzbaren und auch messbaren direkten Wirkungen. Diesen gegenüber können indirekte Wirkungen, wie in Folge der Nutzung hergestellter Produkte, zum Zeitpunkt der Antragstellung nicht ermittelt werden. Sie hängen zudem von mehreren unsicheren Faktoren ab, wie dem Erfolg der Produkte am Markt, dem Produktabsatz und der Art und Weise der Nutzung durch Kunden.

Bei der Bewertung von Produkten besteht die Möglichkeit, dass die Produkte eines geförderten Unternehmens von einem anderen, ebenfalls geförderten Unternehmen erworben und die Produktwirkungen somit erneut gewertet werden. Produktwirkungen in die Bewertung einzubeziehen, birgt daher auch die Gefahr der Mehrfachbewertung derselben Wirkungen, was auszuschließen ist.

Projekte mit so unterschiedlichen Wirkungsarten wie die der Beispielfälle müssen dementsprechend mit spezifischen Verfahren bewertet werden. Die Bewertungskonzeption trägt dem Rechnung, indem die beiden Projektkategorien „investiv“(Beispiel Unternehmen) und „nicht-investiv“ (Beispiel Cluster) gebildet wurden, denen sich alle Vorhaben des Operationellen Programms zuordnen lassen.

Für investive und nicht investive Projekte wurde je ein Indikatorenkatalog entwickelt. Bei den nicht-investiven Maßnahmen werden, wie oben beschrieben, die indirekten Wirkungen als wesentlich be- 
trachtet und dementsprechend als Bewertungsgrundlage herangezogen. Bei den investiven Maßnahmen werden aus den dargelegten Gründen nur die direkten Umweltwirkungen bewertet. $\mathrm{Zu}$ den nicht-investiven Projekten zählen neben dem Clustermanagement die Förderung von Personal- und Sachmitteln bei Projekten in den Bereichen Netzwerke, Forschung und Wissenstransfer. An investiven Projekten werden wie im Beispiel gewerbliche Investitionsvorhaben sowie Investitionen in kommunale Infrastrukturen wie zum Beispiel Technologie- oder Gründerzentren und Forschungsinfrastrukturen gefördert. Beispiele für Letztere sind Laboreinrichtungen oder verfahrenstechnische Versuchsanlagen.

Während einer Testphase wurden Erfahrungswerte mit der Bewertungskonzeption gesammelt, die Anlass gaben, die Systemgrenzen in Bezug auf direkte und indirekte Wirkungen investiver Projekte weiter zu präzisieren. So sind neben den im Beispiel genannten Produktwirkungen weitere, außerhalb geförderter Unternehmen auftretende Projektwirkungen denkbar, die die gesamten Umweltwirkungen mehr oder weniger stark beeinflussen.

Im Fall von Maschineninvestitionen treten Umweltwirkungen an allen Stufen des Lebenszyklusses der Maschinen auf - von der Gewinnung von Ausgangsstoffen für Produktion von Maschinenteilen bis hin zur Entsorgung oder Wiederverwertung. Es konnte jedoch keine solide methodische Grundlage entwickelt werden, um diese und weitere außerhalb des Hofoder Werkstors liegenden Wirkungen mit vertretbarem Aufwand im Rahmen des Antragsverfahrens zu erheben und sie gegenüber den vor Ort im geförderten Unternehmen entstehenden Wirkungen angemessen zu berücksichtigen.

In Anwendung des Hoftor-Prinzips werden daher nur solche Umweltwirkungen bewertet, die vor Ort beim Antragsteller entstehen und die auf Betrieb und Nutzung der Investitionsobjekte im geförderten Unternehmen zurückgehen.

Die Gesamtbewertung der Projekte erfolgt bei investiven und nicht-investiven Projekten jeweils auf einer vierstufigen
Skala von negativ (-1) bis besonders fördernd (+2), wie in Tabelle 1 dargestellt.

Auf Ebene der einzelnen Fragen können Projektwirkungen als „negativ“(-1 Punkt), „neutral“ (0), „positiv“ (+1) oder „sehr positiv“ (+2) angegeben werden. Bei einzelnen Fragen können davon abweichend für besonders positive Umweltwirkungen mehr Punkte vergeben werden.

Mit Bildung der zwei Projektkategorien werden die Vielfalt des Förderprogramms und unterschiedliche Arten von Umweltwirkungen systematisiert und damit eine weitgehende Vergleichbarkeit von Ergebnissen der Projektbewertungen angestrebt. Während der Programmumsetzung werden die Daten zu Umweltbewertungen der Einzelprojekte ausgewertet, dabei nach verschiedenen Förderbereichen und Ebenen des Programms aggregiert und etwa zu Vergleichen von Umweltwirkungen des Mitteleinsatzes nach Förderbereichen genutzt.

\section{Bewertung nicht-investiver Vorhaben}

Die indirekten Umweltwirkungen der oben beschriebenen nicht-investiven Maßnahmen können nur näherungsweise beschrieben und abgeschätzt werden. Daher sind die Fragen zu Umweltwirkungen auf verbal-argumentative Beschreibung indirekter Umweltwirkungen ausgerichtet. Der Indikatorenkatalog enthält vier Fragen, die von Antragstellern mit ausführlichen Erläuterungen zu beantworten und in Tabelle 2 zu sehen sind.

\section{Bewertung von Investitionsvorhaben}

Zur Bewertung investiver Maßnahmen wurde ein Katalog mit insgesamt zwölf Fragen zu umweltrelevanten Projektwirkungen entwickelt, der mit qualitativen und teils quantitativen Angaben zu Umweltwirkungen $\mathrm{zu}$ beantworten ist.

Positiv gewertet werden sowohl absolut als auch relativ verringerte Umweltbelastungen, wie Effizienzsteigerungen in Folge der Projektdurchführung. So wird bei Indikator 2 „Energieeinsatz“ als „positiv“ (1 Punkt) gewertet, wenn sich infolge des
Projekts eine absolute Einsparung von Energie oder eine Steigerung der Energieeffizienz ergibt.

Bei den Fragen zum Ressourcenschutz wird die Quantifizierung positiver Umweltwirkungen mit einem Zusatzpunkt, der zur Bewertung „sehr positiv“ (2 Punkte) führt, quasi belohnt. Voraussetzung für die Angabe von Zahlenwerten zu positiven Wirkungen ist das Wissen der Antragsteller über umweltrelevante Projektwirkungen, das zugleich ein wichtiger Faktor für Umweltverbesserungen ist. Beispiel einer quantifizierten Angabe ist die Verringerung des Materialeinsatzes eines Fertigungsprozesses mithilfe neuer Maschinen um 30 Prozent. Zusatzpunkte werden unabhängig von der Größenordnung der Angaben vergeben.

\section{Ausblick}

Das Verfahren ist seit 2009 als erste praxisnahe Konzeption zur Umweltbewertung EFRE-geförderter Maßnahmen in Baden-Württemberg im Einsatz. Derzeit wird die Konzeption anhand von Fallstudien validiert. Darauf aufbauend werden weitere Schritte zur Verbesserung und Weiterentwicklung der Konzeption unternommen.

\section{Anmerkung \\ Indikatorenformulare mit den Fragenkatalogen und weitere Informationen zum Operationel- len Programm stehen unter http://www.rwb- efre.baden-wuerttemberg.de/ zur Verfügung.}

I AUTOR + KONTAKT

Dominic Kaiser arbeitet als Umweltbeauftragter für das Operationelle Programm RWB-EFRE 2007-2013 Baden-Württemberg in der Verwaltungsbehörde.

Dominic Kaiser, Ministerium für Ländlichen Raum und Verbraucherschutz, Kernerplatz 10, 70182 Stuttgart. Tel.: +49 711 126-2056, E-Mail: dominic.kaiser@mlr.bwl.de 


\section{Lizenzhinweis}

Die Beiträge in ÖkologischesWirtschaften werden unter der Creative-Commons-Lizenz "CC 4.0 Attribution Non-Commercial No Derivatives" veröffentlicht. Im Rahmen dieser Lizenz muss der Autor/Urheber stets genannt werden, das Werk darf nicht bearbeitet, abgewandelt oder in anderer Weise verändert und außerdem nicht kommerziell genutzt werden. Die digitale Version des Artikels bleibt für zwei Jahre Abonnent/innen vorbehalten und ist danach im Open Access verfügbar. 